\title{
Performance of next-gen sequencing platforms in diagnosing autosomal dominant polycystic kidney disease
}

Hamad Ali

Fahd Al-Mulla

Naser Hussain

Medhat Naim

Akram M. Asbeutah

Ali Al Sahow

Mohamed Abu-Farha

Jehad Abubaker

Ashraf Al Madhoun

Sajjad Ahmad

Peter C. Harris

\section{Video Abstract}

Keywords: Scientific Reports, kidneys, DNA, PKD1, clinical utility, whole exome sequencing, WES, genetic diagnosis, genetic analysis, autosomal dominant polycystic kidney disease, ADPKD, renal disease, cysts, NGS, pseudogenes, ultrasonography, renal function tests, mutation screening, human genome project, targeted sequencing

Posted Date: September 23rd, 2019

DOI: https://doi.org/10.21203/rs.2.14979/v2

License: (c) (i) This work is licensed under a Creative Commons Attribution 4.0 International License. Read Full License 


\section{Abstract}

Genetic sequencing is faster and cheaper than ever. But are the latest techniques more reliable than traditional ones? Scientists at the Dasman Diabetes Institute and Kuwait University are investigating that question for one of the trickiest genetic diseases to diagnose: autosomal dominant polycystic kidney disease. ADPKD is an inherited disease in which clusters of fluid-filled cysts accumulate in both kidneys, leading to increased kidney volume, impaired kidney functions, and, ultimately, kidney failure. In fact, ADPKD is the fourth leading cause of kidney failure, affecting one in every 800 to 1000 people worldwide. People with ADPKD may also develop cysts in the liver and other complications. The cause: mutations in genes PKD1 and PKD2. Genetic diagnosis allows doctors to detect the disease before symptoms even arise. The gold standard for doing so is Sanger sequencing. This technique sequences one DNA fragment at a time to detect mutations in the genome. Despite its high accuracy, though, this turnstile process tends to be time-consuming and expensive. That's where so-called next-generation sequencing techniques come in. These methods run multiple fragments simultaneously to sequence the entire genome or only the regions that actually encode for proteins-the exome-in a matter of hours. So why haven't clinicians made the switch? The PKD1 gene, it turns out, is highly deceptive. PKD1 lies in a segmentally duplicated region, where the first 32 coding segments are replicated 6 times around the original gene. These PKD1 look-alikes, or pseudogenes, can influence the sensitivity and reliability of generated results. For the first time, researchers closely examined the potential for different next-gen techniques to replace Sanger sequencing in diagnosing ADPKD. The team sequenced genetic material from 51 patients diagnosed with or at risk for ADPKD using popular techniques, each more targeted than the last: whole-genome sequencing, whole-exome sequencing, and targeted enrichment. Whole-exome sequencing performed the worst-catching mutations in the duplicated part of PKD1 with a sensitivity of just over $7 \%$. Whole-genome sequence, which casts a wider genetic net, produced better results but showed limitations for extensive deletion mutations. Target enrichment performed the best. This regionspecific approach offered the best coverage of the most problematic stretch of PKD1-appearing to overcome the limitations of the other approaches while maintaining the benefits of low cost and fast turnaround. That suggests that next-generation sequencing could be widely utilized in clinical set-ups for diagnosing ADPKD. Getting there, however, will take some work. Whole-exome sequencing in particular could use a boost in read depth, quality, and sensitivity. Such enhancements would allow for more rapid and accurate genetic analysis of ADPKD patients and, ultimately, better disease management and understanding of the molecular pathology underlying this disease. 